\title{
Búsqueda de información electrónica: habilidad necesaria pero poco reconocida.
}

Search of electronic information: Needed but poorly recognized skill.

\section{Sr. Editor:}

La presente es para hacer llegar mi estima a su revista y en especial a los autores del artículo titulado "Encuesta virtual sobre uso de Internet y medios informáticos entre psiquiatras y residentes de psiquiatría en el Perú"(1). Ellos discuten un tema pertinente como son los conocimientos y habilidades básicos que requieren los profesionales de salud, en este caso el uso de las Tecnologías de Información y Comunicación (TICs) para su continua autocapacitación.

Las Tecnologías de información y Comunicación (TICs) en los últimos años se han extendido a un mayor número de usuarios de manera vertiginosa. El acceso al servicio de Internet a nivel nacional llega a 793157 hogares (2) siendo en Lima Metropolitana el $23,4 \%$ de los hogares (3). La expansión de las TICs ha facilitado la accesibilidad y su uso en los hogares peruanos. Así mismo, la autocapacitación por vía electrónica es una forma útil y rápida para acceder a información actualizada, sobre todo en lugares más remotos.

Si bien el artículo trata de un tema de interés quisiera hacer algunos comentarios que podrían aplicarse para mejorar en futuras publicaciones.

La encuesta por vía electrónica es una herramienta muy útil que permite disminuir el gasto de recursos valiosos como son el tiempo y personal dedicados a la recolección de datos. A pesar de que su valor ha sido demostrado en múltiples ocasiones, creo que en este caso específico fue un error utilizarla debido al importante sesgo que esta puede presentar en los resultados (4). Al preguntarle a personas por vía electrónica acerca de su nivel de habilidad en el uso de herramientas electrónicas y características asociadas, el resultado se sesga de manera importante hacia una mayor habilidad. Un ejemplo de esto sería el detener a un conductor, transitando en una avenida, $\mathrm{y}$ preguntarle si sabe manejar.

Asimismo, creo que es difícil que la muestra sea representativa de la realidad nacional debido a la limitada selección y respuesta de los participantes elegidos (5). El gran porcentaje de rechazo posiblemente represente a la gran cantidad de personas que no tiene acceso continuo, o interés en dicho acceso (al Internet); nuevamente sesgando sus resultados hacia profesionales de centros urbanos con mayor habilidad y tiempo de uso con esta herramienta.

Con respecto al tiempo de uso, me parece que las distribuciones usadas no eran normales, considerando su número y las amplias desviaciones estándar (DE) descritas. Esto se puede observar en el caso de la opción "Psiquiatra con subespecialidad", de la tabla 3: "Categoría profesional y genero versus tiempo (horas por semana) de uso de Internet", en la que la media es de 13,7 horas a la semana y la DE de 13,7 horas igualmente. Haciendo que algunos de sus análisis bivariados puedan tener resultados diferentes.

Entre las "bases de datos científicas" elegidas, destaca la separación que hacen entre Medline y Pubmed. Si bien es posible que se considere el uso de Medline de una manera más amplia, creo que en el caso del presente estudio desean representar lo mismo por lo que deberían considerarse una sola opción. Asimismo, HINARI no cuenta con un buscador de información independiente por lo que también usa al Pubmed para la búsqueda de artículos. De las fuentes de información casi todas permiten la búsqueda de artículos científicos de diversa calidad, con excepción de UpToDate que está enfocado a "artículos de revisión" (6).

En general, me parece que el tema tratado es muy interesante e importante. Se deben evaluar las habilidades de los profesionales de salud para implementar mejoras en su desempeño, tales como intervenciones de capacitación en el uso de estos medios 
o un mejor, o más amplio, acceso a la información pertinente. La información presentada en el artículo es útil como una línea base de investigación, pero se deben realizar nuevos estudios, que nos permitan obtener información más detallada para evaluar dichas intervenciones.

\section{Jorge Osada ${ }^{1}$}

\section{Correspondencia:}

Jorge Osada

Alfredo Salazar 472 dept 201,

San Isidro, Lima, Perú.

Correo electrónico:j_osada@hotmail.com

Celular: 51992304701

\section{REFERENCIAS BIBLIOGRÁFICAS}

1. Herrera-Lopez VE, Stucchi-Portocarrero S, Favio Vega-Galdós F. Encuesta virtual sobre uso de Internet y medios informáticos entre psiquiatras y residentes de psiquiatría en el Perú. Rev Neuropsiquiatr. 2011; 74(1): 191-99.

2. OSIPTEL - Organismo Supervisor de Inversión Privada en Telecomunicaciones. Evolución anual del número de suscriptores del servicio de acceso a Internet fijo según tecnología de acceso. Lima: INICTEL. URL disponible en: http://www.osiptel.gob.pe/ WebsiteAjax/WebFormgeneral/sector/ wfrm_Consulta_Informacion_Estadisticas. aspx? CodInfo $=13475 \& \mathrm{CodSubCat}=864 \& \mathrm{Ti}$ tuloInformacion $=6 . \% 20$ Indicadores $\% 20 \mathrm{de} \% 20$ Internet\&DescripcionInformacion (Fecha de acceso: 20 de enero del 2012).

3. Instituto Nacional de Estadística e Informática. Nota de prensa. $\mathrm{N}^{\circ} 086$ - 028 Junio 2010. Lima: INEI. URL disponible en: http://www.inei.gob.pe/web/ NotaPrensa/Attach/10854.pdf (Fecha de acceso: 20 de enero del 2012).

4. Hulley SB, Martin JN, Cummings SR. Planificación de las mediciones: precisión y exactitud. En: Hulley SB, Cummings SR, Browner WS, Grady DG, Newman TB. Diseño de investigaciones clínicas. Barcelona: Wolters Kluwer Health España, Lippincott Williams \& Wilkins; 2008. p. 43-56.

5. Hulley SB, Newman TB, Cummings SR. Elección de los participantes en el estudio: especificación, muestreo y reclutamiento. En: Hulley SB, Cummings SR, Browner WS, Grady DG, Newman TB. Diseño de investigaciones clínicas. Barcelona: Wolters Kluwer Health España, Lippincott Williams \& Wilkins; 2008. p. 31-42.

6. UpToDate Incorporated. About UpToDate. URL disponible en: http://www.uptodate.com/home/about/ index.html (Fecha de acceso: 10 de diciembre del 2011).

1. Médico-Epidemiólogo.Grupo de Trabajo en Salud Mental, Universidad Peruana Cayetano Heredia. Lima, Perú.

\section{Réplica}

\section{Reply}

\section{Sr. Editor:}

Agradecemos al Dr. Osada por los comentarios hechos a nuestro artículo, lo que muestra el interés en el tema y contribuye de forma valiosa al enriquecimiento del estudio realizado.

Reconocemos, tal y como lo establecimos en la misma discusión del artículo, que efectivamente puede haber un sesgo en el estudio respecto al uso de una encuesta virtual que no llegue a todos los colegas de la especialidad, por las limitaciones de uso del
Internet $\mathrm{y} / \mathrm{o}$ registro en la base de datos de correos. Los autores elegimos dicha opción como herramienta útil y práctica, considerando la flexibilidad de su diseño, el ahorro de tiempo y el factor económico, teniendo como base los datos registrados virtualmente en el grupo de correos de la Asociación Psiquiátrica Peruana. Es posible que los encuestados que utilizaron la vía electrónica tuvieran una mayor familiaridad con el uso de recursos virtuales y no fueran totalmente representativos de la población objetivo, pero esto permitió el tener respuestas de psiquiatras del interior del país, lo que hubiera sido más difícil con encuestas impresas. Pese a todo, en nuestra opinión, el sesgo mencionado no compromete los resultados para el objetivo que nos planteamos, al ser un estudio inicial exploratorio del tema, situación que también ha sido considerada en investigaciones análogas (1-4). 
El Dr. Osada cuestiona la representatividad de la muestra según la realidad nacional y debido a una aparente limitada selección y respuesta de los participantes elegidos. Sin embargo, hay que mencionar que en el presente estudio descriptivo la muestra predominante ubicó a los encuestados en Lima, lugar donde ejercen mayoritariamente los médicos psiquiatras en la actualidad (aproximadamente el $85 \%$ del total) coincidiendo con la distribución real en nuestro país. Debe considerarse además que la tasa de respuestas para encuestas electrónicas reportada en otros estudios es del 32,52\% (5), cifra inclusive menor que la obtenida por nosotros, que fue del $35 \%$. Cabe señalar por otro lado que la base de datos de la Asociación Psiquiátrica Peruana contaba con aproximadamente el $70 \%$ de psiquiatras registrados a nivel nacional en la fecha de ejecución del estudio.

En relación a la desviación estándar de la variable tiempo de uso, cabe mencionar que la amplitud de la misma puede ser debida a que no tenga una distribución normal, pero también-y más probablemente- a la dispersión existente en la misma variable, lo cual es un hecho esperable. La preocupación sobre la distribución normal sería importante en el caso que se quisiera validar una prueba de hipótesis con la intención de extrapolar los resultados a todos los psiquiatras a través de intervalos de confianza, lo cual no fue el objetivo de nuestro estudio.

Finalmente, coincidimos con el Dr. Osada en que el uso de Internet es cada vez más generalizado e influyente en las modalidades de la práctica profesional, lo cual nos motiva a desarrollar una línea de investigación al respecto, que permita valorar el potencial de uso, aspectos éticos, implicancias y cambios en las relaciones del médico psiquiatra con los usuarios de los servicios de salud.

\section{Vanessa Herrera-Lopez ${ }^{1}$, Santiago Stucchi- Portocarrero ${ }^{2}$, Favio Vega-Galdós ${ }^{3}$.}

\section{Correspondencia:}

Vanessa Herrera Lopez

Complejo Habitacional Rumihuasi Dpto 403 Bloque F

La Victoria. Lima, Perú.

Correo electronico: vanessaherrera7@hotmail.com

\section{REFERENCIAS BIBLIOGRÁFICAS}

1. Castells M. Médicos en Internet. URL disponible en http://www.comb.cat/Upload/Documents/ Articulo_medicos_Internet.pdf (último acceso: marzo del 2012).

2. Iantorno G, Corti R, Fernández LM, Soifer L, Bilder C, Schvartz AM, et al. Encuesta piloto virtual a médicos argentinos (usuarios de la red intramed) sobre conocimientos, conductas diagnósticas y terapéuticas referidas a la dispepsia funcional. Acta Gastroenterol Latinoam. 2007; 37 (Suppl 1): 29-53.

3. Kovic I, Lulic I, Brumini G. Examining the medical blogosphere: An online survey of medical bloggers. J Med Internet Res. 2008;10(3):e28

4. Romano M, Gesualdo F, Pandolfi E, Tozzi AE, Ugazio AG. Use of the Internet by Italian pediatricians: habits, impact on clinical practice and expectations. BMC Med Inform Decis Mak. 2012; 12: 23.

5. Hamilton MB. Online survey response rates and times: Background and guidance for industry. Longmont, Colo, Ipathia, Inc. / SuperSurvey. Available at http://www.supersurvey.com/papers/ supersurvey_white_paper_response_rates.pdf (último acceso: setiembre del 2012).

6. Braithwaite D, Emery J, De Lusignan S, Sutton $\mathrm{S}$. Using the Internet to conduct surveys of health professionals: a valid alternative? Fam Pract. 2003; 20: 545-51.

7. Herrera-Lopez VE, Stucchi-Portocarrero S, VegaGaldós F. Encuesta virtual sobre uso de Internet y medios informáticos entre psiquiatras y residentes de psiquiatría en el Perú. Rev Neuropsiquiatr. 2011;74(1):191-99.

\footnotetext{
1. Residente de Psiquiatría. Instituto Nacional de Salud Mental “Honorio Delgado -Hideyo Noguchi”. Lima, Perú.

2. Médico Psiquiatra. Instituto Nacional de Salud Mental "Honorio Delgado -Hideyo Noguchi”. Universidad Peruana Cayetano Heredia. Lima, Perú.

3. Médico Psiquiatra. Instituto Nacional de Salud Mental "Honorio Delgado -Hideyo Noguchi”. Universidad Nacional Mayor de San Marcos. Lima, Perú.
} 